\title{
Determination and Testing the Domination Numbers of Helm Graph, Web Graph and Levi Graph Using MATLAB
}

\author{
Ayhan A. khalil
}

Dept. of Computer Eng. Technology / Technical College of Mosul Foundation of Technical Education

Received

$10 / 02 / 2010$
Accepted

07 / 04 / 2010

\begin{abstract}
الملخص
يقال لأي مجموعة جزئية S من مجموعة الرؤوس V في بيان G بأنها مجموعة مهيمنة (مسيطرة) Dominating set

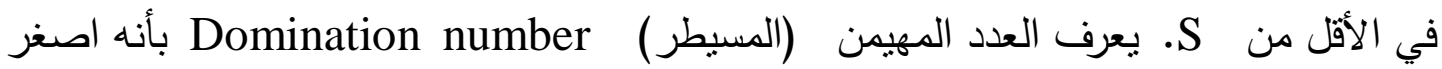

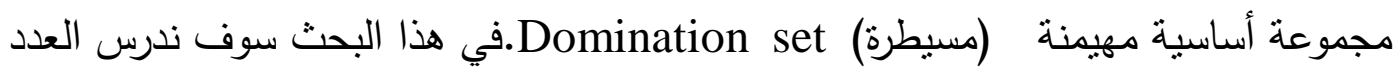

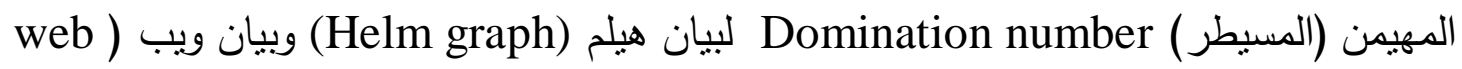

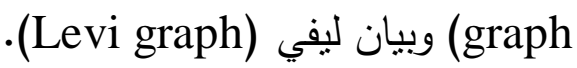
كذللك تأكدنا من النتائج النظرية التي توصلنا إليها بواسطة الحاسبة باستعمال الماتلاب (matlab) $\gamma\left(H_{n}\right)=n$ الرؤوس المهيمنة، حيث توصلنا إلى النتائج التالية: $\gamma\left(W_{m}\right)=m$

$\gamma($ Levi graph $)=10$
\end{abstract}

\begin{abstract}
A set $S \subseteq V$ is dominating set of a graph G, if every vertex in $V-S$ is adjacent to at least one vertex in $S$. The domination number denoted by $\gamma(G)$ is defined to be the minimum cardinality of dominating set in $G$.

We investigate the domination numbers of Helm graph, web graph and Levi graph. Also we testing our theoretical results in computer by introduce a matlab procedure to calculate the domination numbers $\gamma(G)$, dominating set $S$ and draw this graphs that illustrated the vertices of domination this graphs. It is proved that:

$$
\begin{aligned}
& \gamma\left(H_{n}\right)=n \\
& \gamma\left(W_{m}\right)=m \\
& \gamma(\text { Levi graph })=10
\end{aligned}
$$
\end{abstract}


Keywords: Dominating set, Domination number, Helm graph, Web graph Levi graph.

Throughout this paper we consider only finite, undirected and simple graph (i.e. without loops and multiple edges). Our terminology and notations will be standard except as indicated. For undefined terms see [2],[3] and [4].

Let $G=(V, E)$ be a graph, where $\mathrm{V}$ denotes the set of vertices in $\mathrm{G}$ and $\mathrm{E}$ denotes the set of edges in G. For any vertex $v \in V$ the open neighborhood of $\mathrm{v}$ is the set:

$N(v)=\{u \in V \mid u v \in E(G)\}$

and the closed neighborhood

$N[v]=N(v) \cup\{v\}$.

For a set $S \subseteq V$ the open neighborhood is $N(S)=\mathrm{U}_{v \in S} N(v)$ and the closed neighborhood is $N[S]=N(S) \cup S$. A set $S \subseteq V$ is dominating set if $N[S]=V$ or equivalently every vertex in $V-S$ is adjacent to at least one vertex in $\mathrm{S}$. The domination number $\gamma(G)$ is the minimum cardinality of dominating set in $\mathrm{G}$ [1].

In other words we defined the domination number of a graph $\mathrm{G}$ by $\gamma(G)$, to be the order of smallest dominating set of $\mathrm{G}$. A dominating set $\mathrm{S}$ with $|S|=\gamma(G)$ is called a minimum dominating set [8].

Dominating set appear to have their origins in the game of chess where the goal is to cover or dominate various squares of a chessboard by certain chess pieces. The problem of determine domination numbers of graphs first emerged in 1862 of de Jaenisch he wanted to find the minimal number of queens on chessboard, such that every square is either occupied by a queen or can be reached by a queen with single move[4]. Domination can be useful tool in many chemical structures [7] also there is many application of domination theory in wireless communication networks [5] business network and making decisions.

Definition(1): The Helm graph $H_{n}$ is a simple graph obtain from $n$ wheel graph $W_{n}$ by adjoining a pendant edge at each vertices of the cycle $C_{n}$ [9].
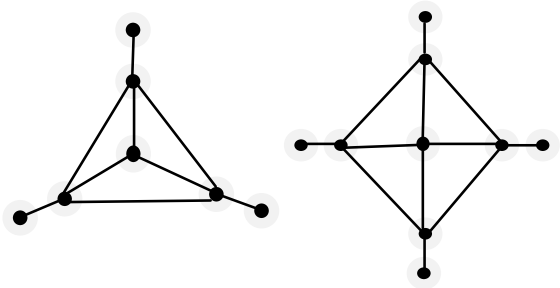

$\mathbf{H}_{3}$

$\mathbf{H}_{4}$

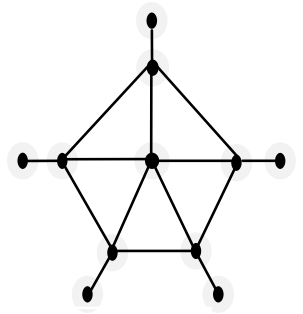

$\mathbf{H}_{5}$

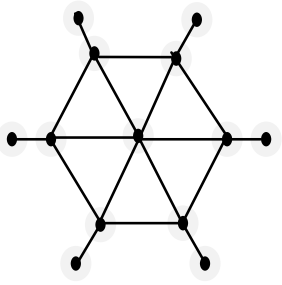

$\mathbf{H}_{6}$

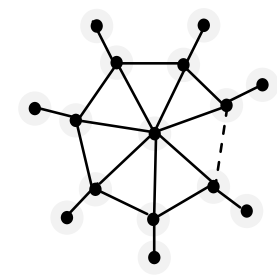

$\mathbf{H}_{n}$

Fig.1: Helm graph 
We first define a prism graph then the Web graph

Definition(2): A prism graph $Y_{m, n}$ is a simple graph given by the Cartesian product graph $Y_{m, n}=C_{m} \times P_{n}$ where $C_{m}$ is a cycle with $m$ vertices and $P_{n}$ is a path with $n$ vertices.

A web graph is a prism graph $Y_{m, a}$ with the edges of the outer cycle removed and denoted by $W_{m}[10]$.

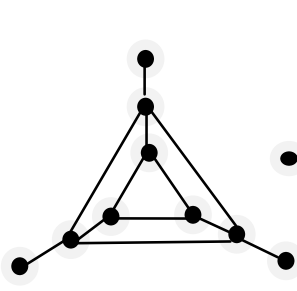

$\mathbf{W}_{3}$

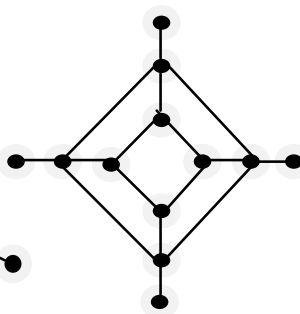

$\mathbf{W}_{4}$

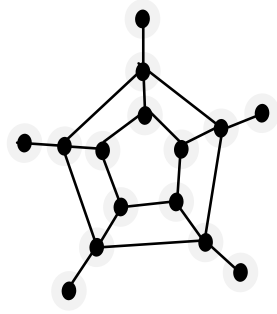

$\mathbf{W}_{5}$

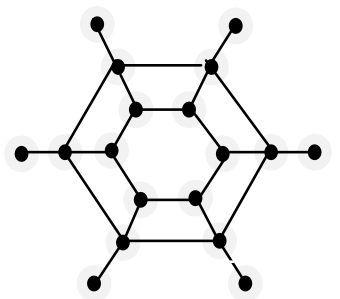

$\mathrm{W}_{6}$

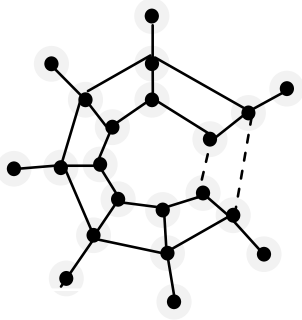

$\mathbf{W}_{\mathrm{m}}$

Fig.2: Web graph

Definition(3): The Levi graph can be written as $G_{1} \cup G_{2}$ where $G_{1}$ and $G_{2}$ are shown in Fig.3 see [11]

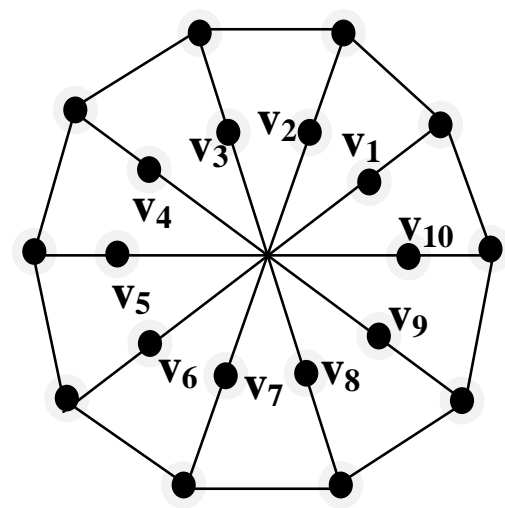

$\mathbf{G}_{1}$

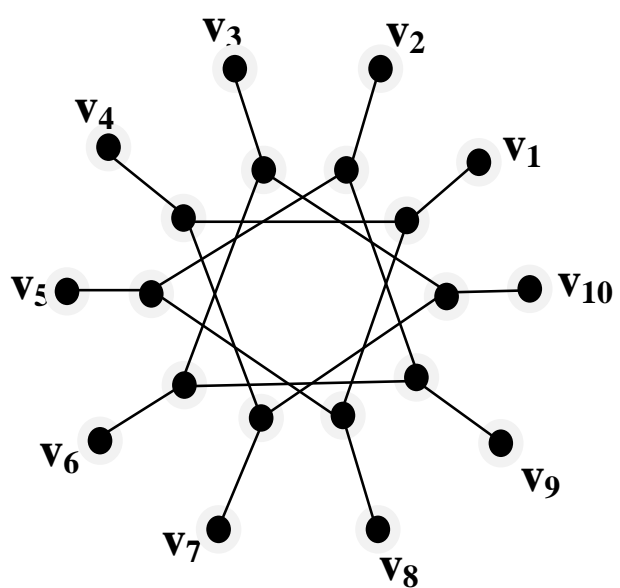

$G_{2}$

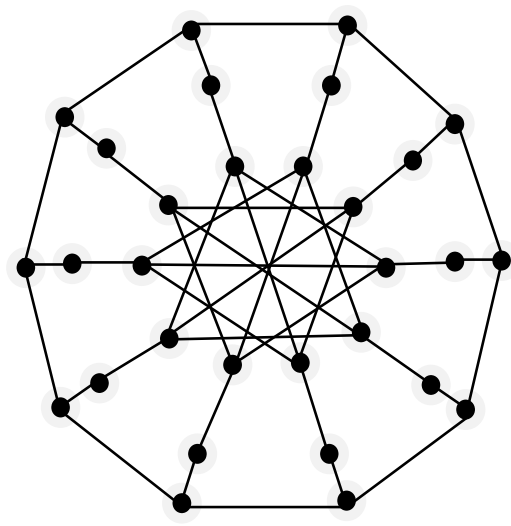

$G_{1} \cup G_{2}=$ Levi graph

Fig.3

The following theorem is useful in our work:

Theorem (1): ([6], p.546)

A dominating set $D$ of a graph $G$ is minimal if and only if for each vertex $v \in D$ one of the following conditions satisfied

(i) There exist a vertex $u \in V-D$ such that $N(u) \cap D=\{v\}$

(ii) $v$ is an isolated vertex in $D$. 


\section{On domination numbers of Helm graph $H_{n}$ :}

Theorem(2): $\gamma\left(H_{n}\right)=n$

Proof: For $n \geq 3$ let the vertices of this graph labeled by:

$V\left(H_{n}\right)=\{1,2,3, \ldots, 2 n+1\}$. (See Fig.4)

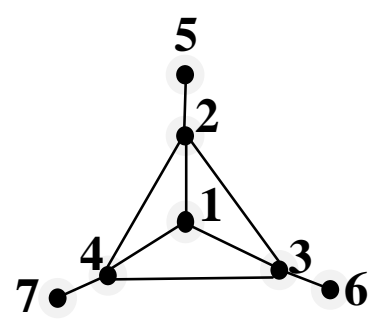

$\mathbf{H}_{3}$

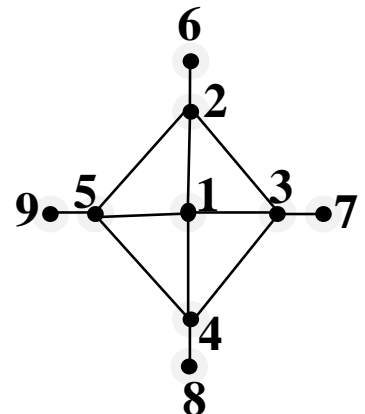

$\mathbf{H}_{4}$

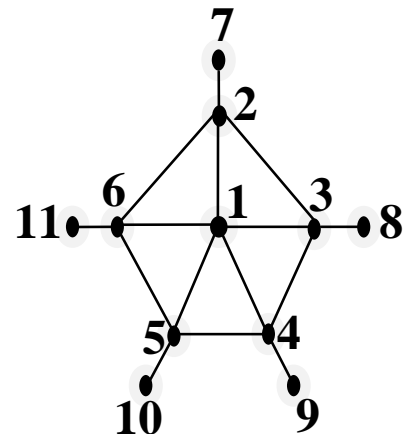

$\mathbf{H}_{5}$

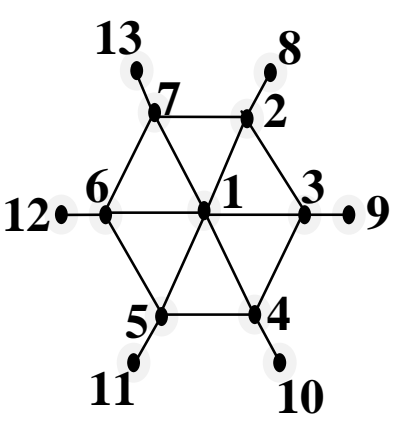

$\mathbf{H}_{6}$

Fig.4

We given the dominating set of this graph by a set isomorphic to $S$

Where $S=\{i \mid i=2,3,4, \ldots, n+1\}$

The minimality of the set $S$ follows from theorem(1) by using the contrary of this theorem.

Assume that $S$ is not minimum dominating set then $\exists v \in S$, such that $S^{I}=S-\{v\}$ is dominating set of $H_{n}$.

Therefore $\forall u \in N[v], \exists k \in S-\{v\}, k \in N[v]$.

Also note that for any vertex $v \in S$ the end points of this graph of the form $(i+n)$ that adjacent to $v$ not dominating with any vertex in $S^{r}$ (i.e. $\left.\left[S^{\prime}\right] \neq V\right)$.

So clearly that $S^{t}$ is not dominating set (by the definition of dominating set).

Chosen $S$ in this way ensures $S$ minimum

$\therefore S$ is minimum

So $\gamma\left(H_{n}\right)=|S|=n$.

On domination number of web graph $W_{m}$ :

Theorem(3): the domination number of web graph is: $\gamma\left(W_{m}\right)=m$

Proof: For $m \geq 3$ let the vertices of this graph labeled by:

$V\left(W_{m}\right)=\{1,2,3, \ldots, 3 m\}$. (See Fig.5) 


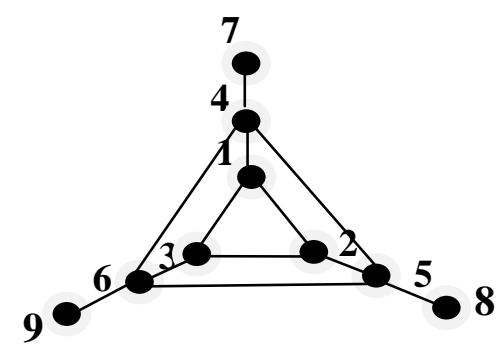

$\mathbf{W}_{3}$

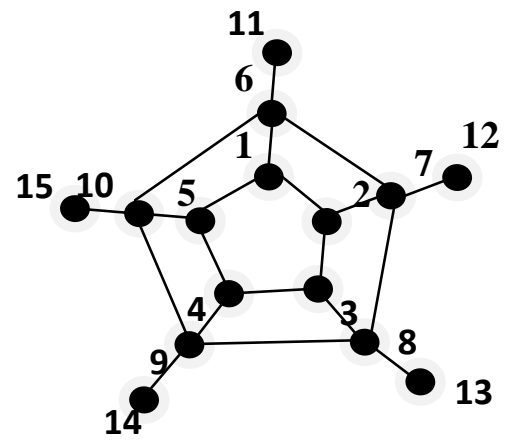

$\mathbf{W}_{5}$

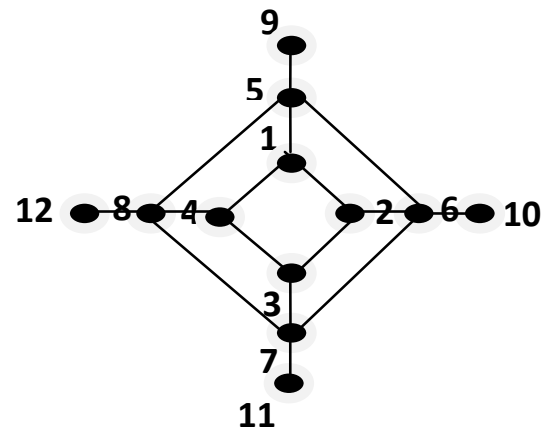

$\mathbf{W}_{4}$

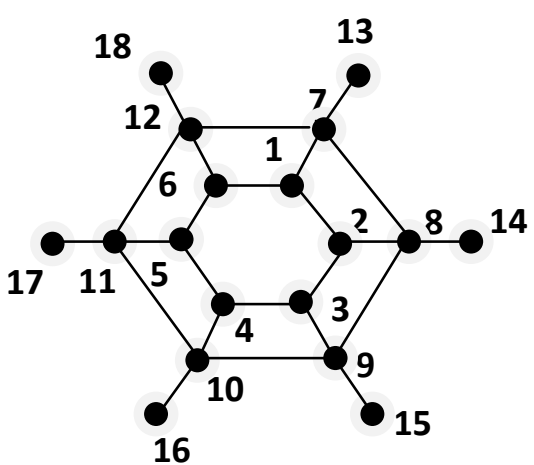

$\mathbf{W}_{6}$

Fig.5

First we given the dominating set of this graph by a set isomorphic to $S$ Where $S=\{i+m \mid i=1,2,3, \ldots, m\}$ the proof of the minimality is similarly in theorem(2)

So we must show that there is no proper subset $S^{I} \subset S$ is dominating set of $W_{m^{*}}$

If $v$ is any vertex in $S$ we have always two vertices of the form $\{i\}$, and $\{i+2 m\}$ not dominating with any vertex in $S^{r}$ so $N\left[S^{\prime}\right] \neq V$

$\therefore S^{r}$ is not dominating set(by definition of dominating set), this ensures $S$ is minimum.

$\therefore \gamma\left(W_{m}\right)=|S|=m$.

\section{On domination numbers of Levi graph :}

As in definition(3) Levi graph can be written as $G_{1} \cup G_{2}$ where $G_{1}$ and $G_{2}$ shown in Fig.3. So we first determine the domination number of $G_{1}$ and $\mathrm{G}_{2}$ then we determine the domination number of Levi graph.

Lemma(1): $\gamma\left(G_{1}\right)=7$

Proof: let the vertices of this graph labeled by: 
$V\left(G_{1}\right)=\{i\} \cup\left\{v_{i}\right\}$ where $i \in\{1,2, \ldots, 10\}$. (See Fig.6)

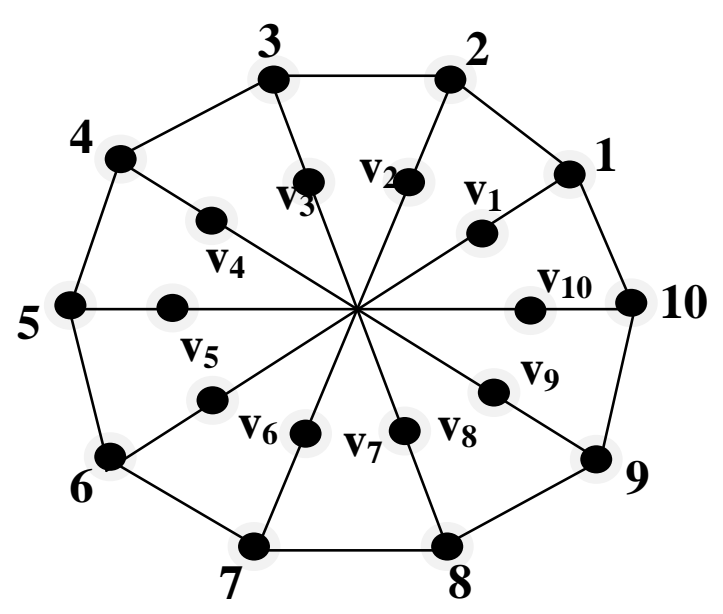

Fig.6: $G_{1}$

We given the dominating set of this graph by a set isomorphic to $S$ Where $=\left\{2,5,7,10, v_{4}, v_{6}, v_{8}\right\}$.

the proof of the minimality is as in theorem(2)

So we must show that there is no proper subset $S^{I} \subset S$ dominating set of $G_{1}$.

For any $v \in S, v$ is one of the following:

$\{2\}$ or $\{5\}$ or $\{7\}$ or $\{10\}$ or $\left\{v_{4}\right\}$ or $\left\{v_{6}\right\}$ or $\left\{v_{8}\right\}$.

We divided the set $S$ into two sets: $S_{1}=\{2,5,7,10\}$ the vertices that is the outer circle and the inner vertices in $S_{2}=\left\{v_{4}, v_{6}, v_{8}\right\}$.

So if $v \in S_{1}=\{2,5,7,10\}$ we have 4 cases:

Case (1): when $v=\{2\}$ we have $S-\{2\}=S^{I}$

$S^{I}$ is not dominating set because there is 3 vertices $\left\{1,3, v_{2}\right\}$ not dominating with any vertex in $S^{I}$. In other words there is 3 vertices in $S-V$ is not adjacent to any vertex in $S-\{v\}$.

Case (2): when $v=\{5\}$ we have $S-\{5\}=S^{I}$.

Also we have 2 vertices $\left\{2, v_{6}\right\}$ in $S-V$ is not adjacent to any vertex in $S-\{v\}$

$\therefore S^{r}$ is not dominating set.

Case (3): when $v=\{7\}$ we have $S-\{7\}=S^{r}$.

Also we have $\left\{v_{7}\right\}$ a vertex in $S-V$ is not adjacent to any vertex in $S-\{v\}$

$\therefore S^{r}$ is not dominating set.

Case (4): when $v=\{10\}$ we have the same case in case (2).

There is 2 vertices $\left\{9, v_{10}\right\}$ in $S-V$ is not adjacent to any vertex in $S-\{v\}$. 
Now, similarly if $v \in S_{2}$ we have at least one vertex of the form $\left\{v_{i+5} \mid i=4,6,8\right\}(\bmod 10)$ not adjacent to any vertex in $S-\{v\}$.

So chosen $\mathrm{S}$ in this way ensures that there is no proper subset of $S$ dominating $G_{I}$

$\therefore S$ minimum

$\gamma\left(G_{1}\right)=|S|=7$.

Lemma(2): $\gamma\left(G_{2}\right)=10$

Proof: let the vertices of this graph labeled by:

$V\left(G_{1}\right)=\{i+10\} \cup\left\{v_{i}\right\}$ where $i \in\{1,2, \ldots, 10\}$. (See Fig.7)

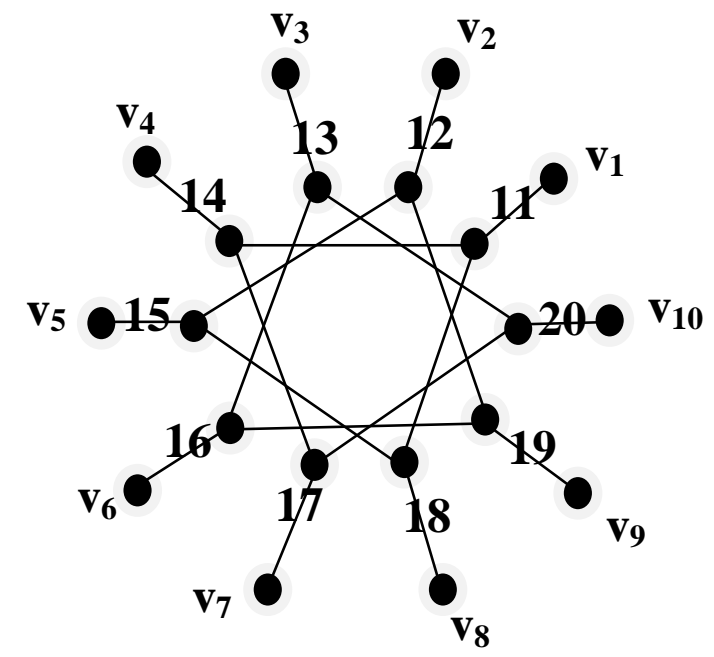

Fig.7: $G_{2}$

We given the dominating set of this graph by a set isomorphic to $S$.

Where $S=\{i+10\}$ where $i \in\{1,2, \ldots, 10\}$ the inner vertices

It is easy to see that $S$ minimum since if $S$ is not minimum then there is proper subset $S^{I} \subset S$ is dominating set.

if $v$ any vertex in $S$, so $S^{I}=S-\{v\}$ for every vertex $v$ the vertices which is refer to the end points of this graph not adjacent to any vertex in $S^{T}$.

$\therefore S^{r}$ is not dominating set

$\therefore S$ is minimum

$\gamma\left(G_{2}\right)=|S|=10$.

Theorem(4): $\gamma$ (Levi graph) $=10$

Proof: The union of the graph $G_{1}$ in lemma (1) and $G_{2}$ in lemma (2) know as Levi graph; has (30) vertices labeled as same in lemma(1) and lemma(2). (See Fig.8) 


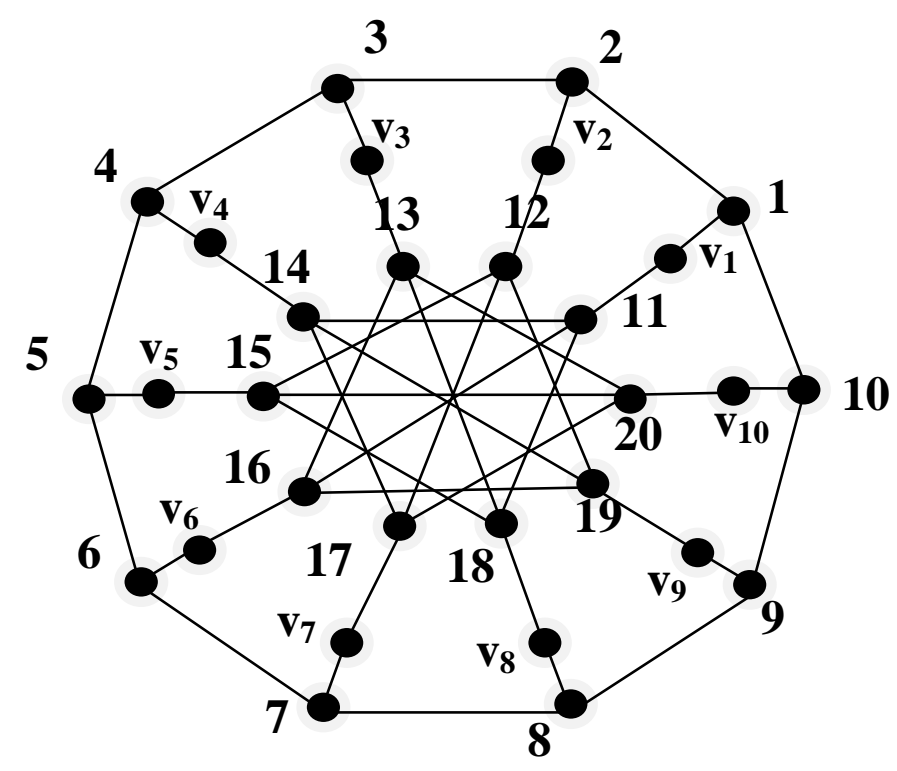

Fig.8

We given the dominating set of this graph by a set isomorphic to $S$.

Where $=\{2,3,5,6,9,10,11,14,17,18\}$.

Also the proof of the minimality is as in theorem(2), assume $S^{I} \subset S$ is dominating set of Levi graph.

We divided the set $S$ into two sets: $S_{1}=\{2,3,5,6,9,10\}$ the vertices that is in the outer circle and $S_{2}=\{11,14,17,18\}$.

If $v \in S_{1}=\{2,3,5,6,9,10\}$ we have the vertices in the form $\left\{v_{i} \mid i=1,2, \ldots, 10\right\}$ not dominating with any vertex in $S-\{v\}$.

Similarly If $v \in S_{2}$ we have the vertices in the form $\{\hat{j} \mid j=1,2, \ldots, 10\}$ not dominating with any vertex in $S^{I}$.

$\therefore S^{f}$ is not dominating set

$\therefore S$ is minimum

$\gamma\left(G_{1} \cup G_{2}\right)=|S|=10$.

Determination and testing the domination numbers of Helm graph and web graph using matlab:

In this paper we writing a programs in matlab for the purpose of making sure and certainty of the validity of our theoretical results for compute the dominating sets $S$ and the domination number of Helm graph ,Web graph and the union of two special graphs $G_{1}$ and $G_{2}$. Moreover we draw this results in the same graph by referring to the vertices that domination this graphs with different color from the other vertices (which colored black). We compute examples for special cases of Helm graph and the Web graph then generalization the results for any $(n)$ of Helm graph and for any $(m)$ for Web graph because in every execution of this programs request an input $n$ or $m$ for this two graphs. 
For the domination numbers of Helm graph $H_{n}$ :

By the program below we demonstrate how to find the domination number of Helm graph $\boldsymbol{H}_{\boldsymbol{n}}$ using matlab:

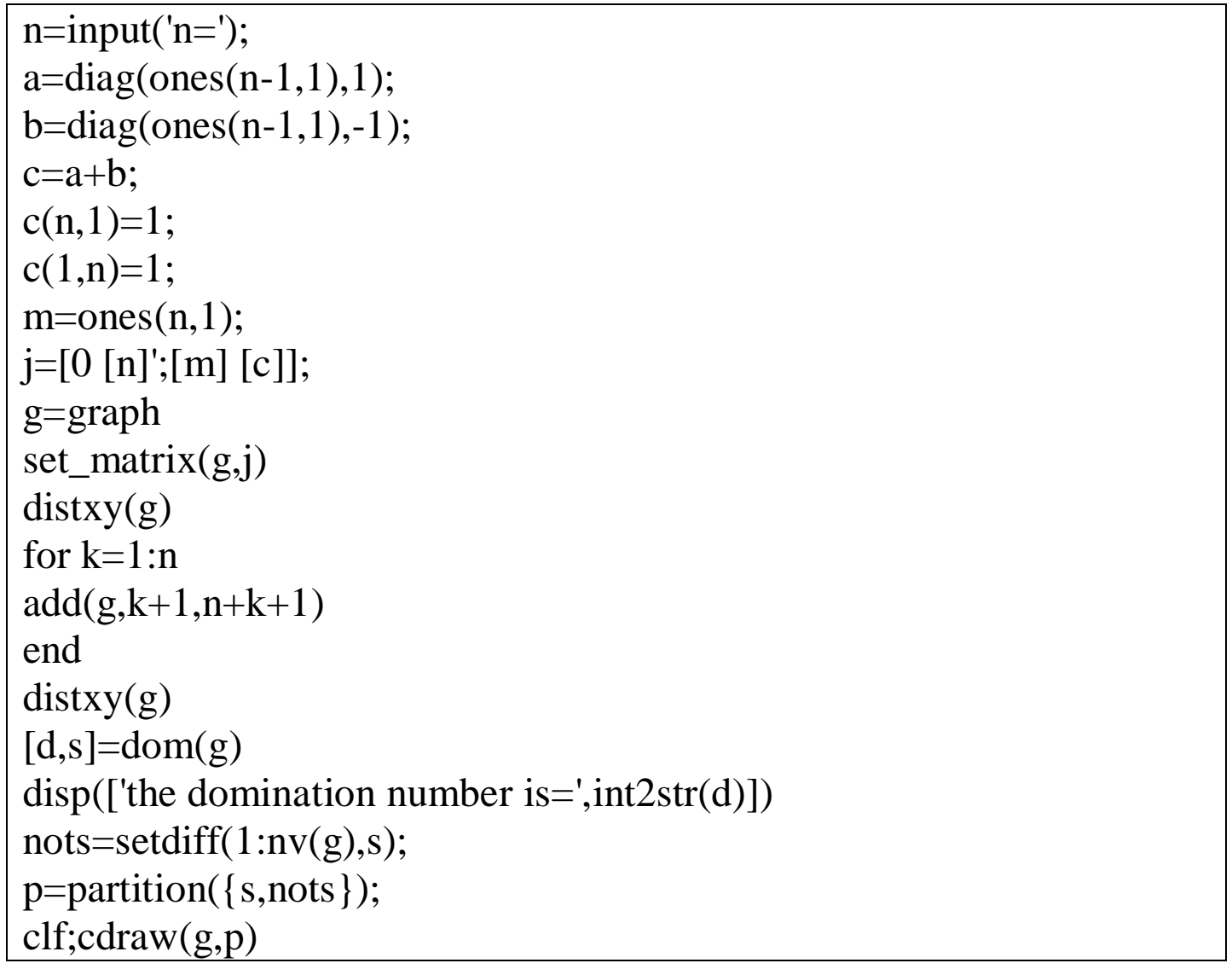

Fig.(9): explain the particular results of the domination number of Helm

For $\mathbf{n}=3$

For $n=4$
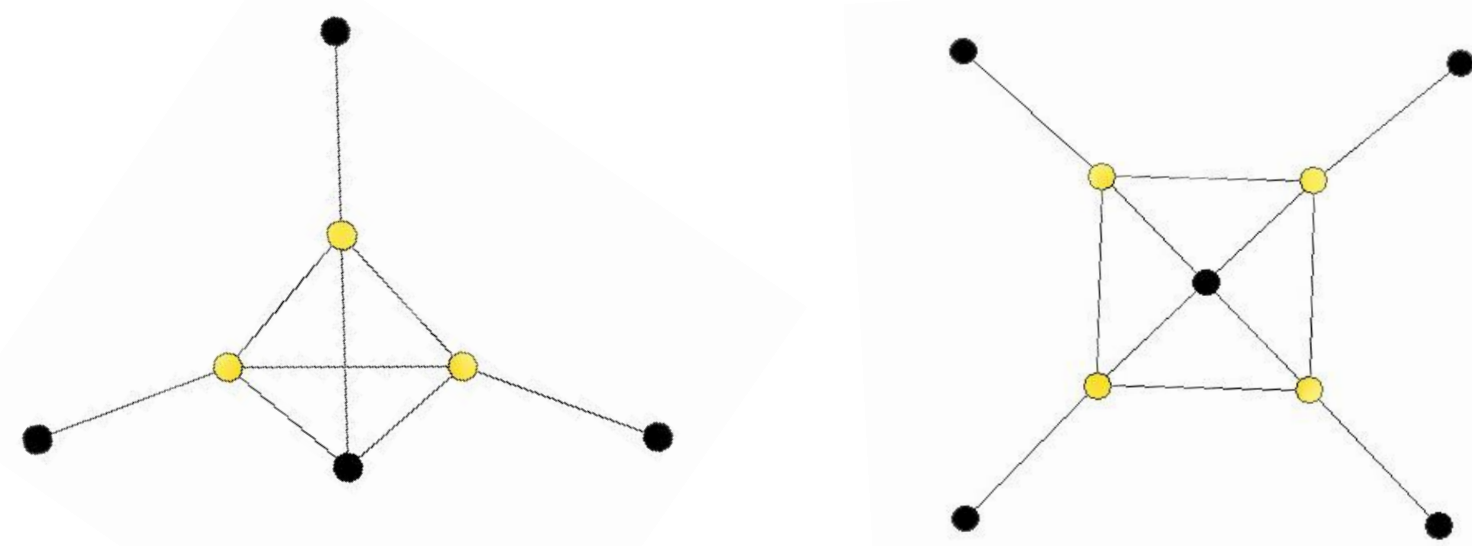

$\mathbf{H}_{3}$

$\mathbf{H}_{4}$ 
For $\mathbf{n}=5$

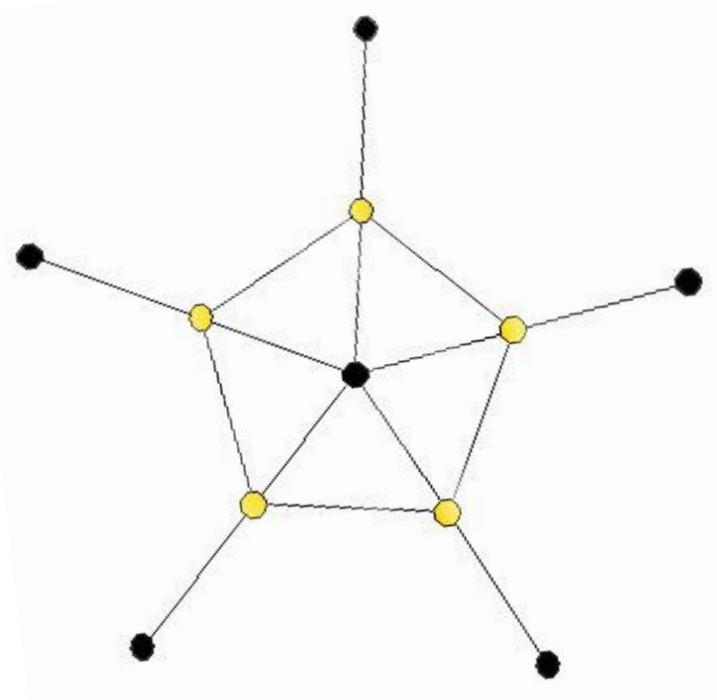

$\mathbf{H}_{5}$
For $n=6$

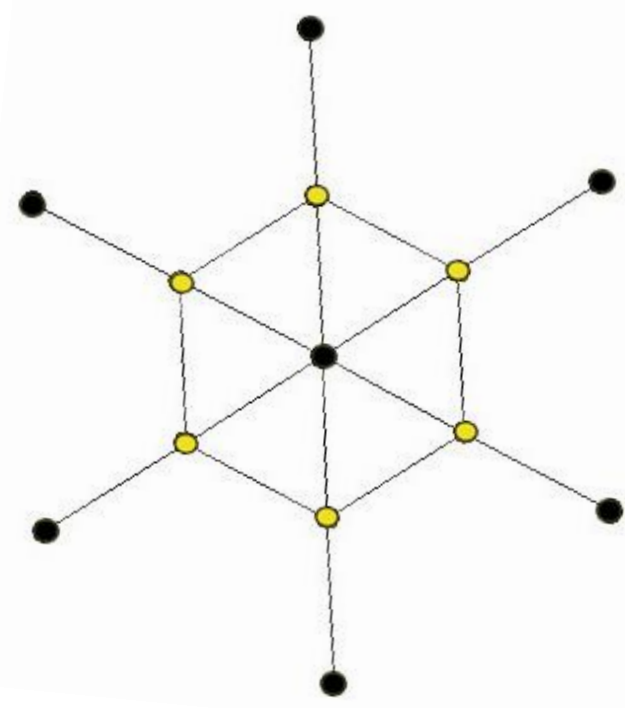

$\mathbf{H}_{6}$

Fig.(9)

For the domination numbers of Web graph $W_{m}$ :

By the program below we demonstrate how to find the domination number of Web graph $\boldsymbol{W}_{m}$ using matlab:

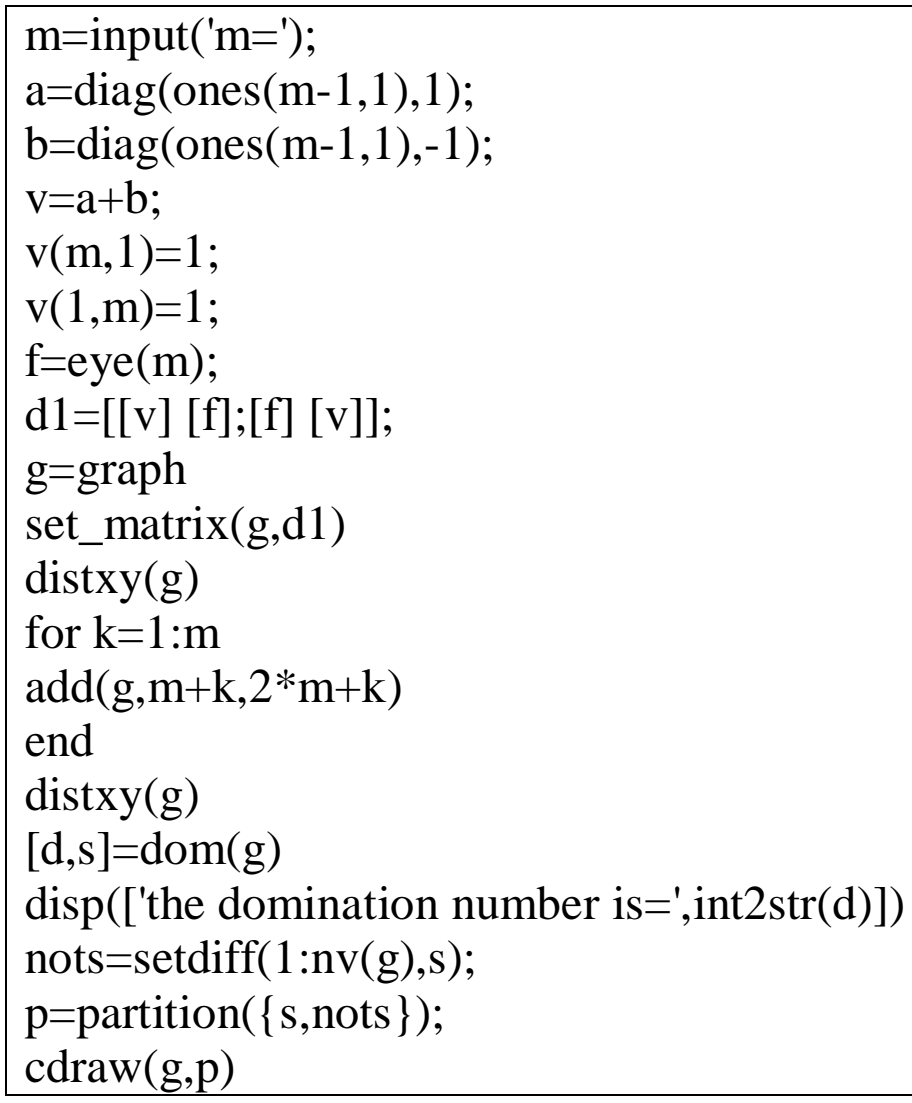


And Fig.(10) explain the particular results of the domination number of Web graph.

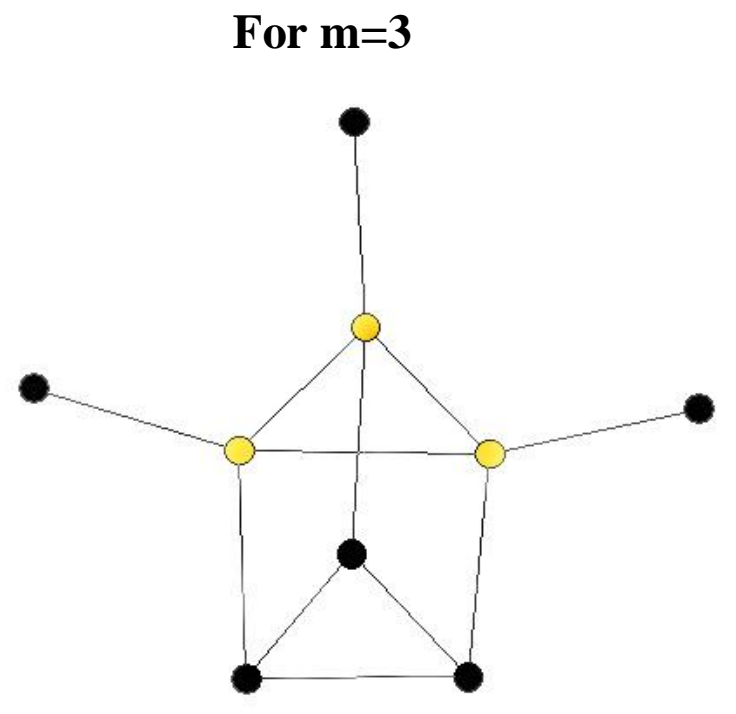

$\mathbf{W}_{3}$

For $m=5$

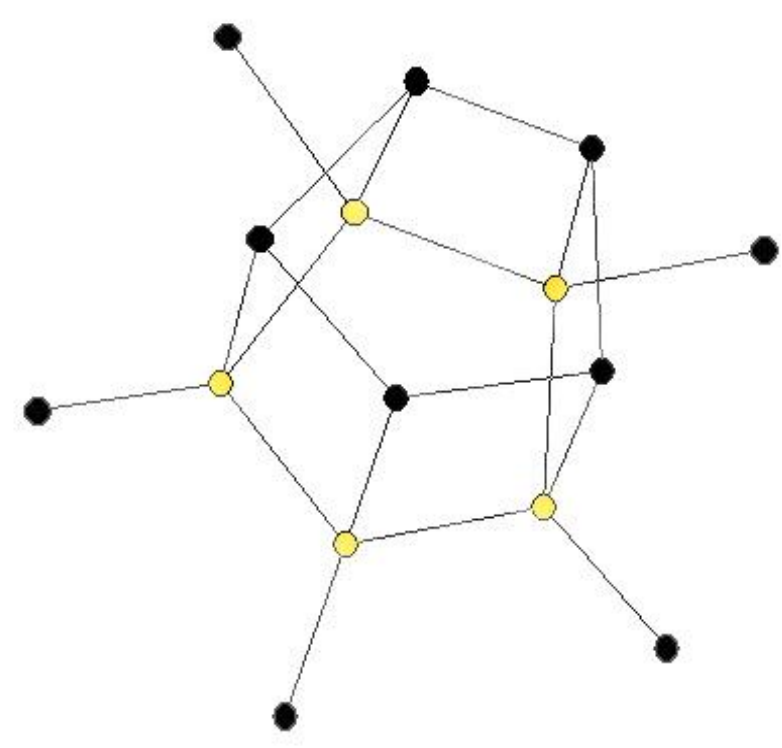

$\mathbf{W}_{5}$
For $\mathbf{m}=4$

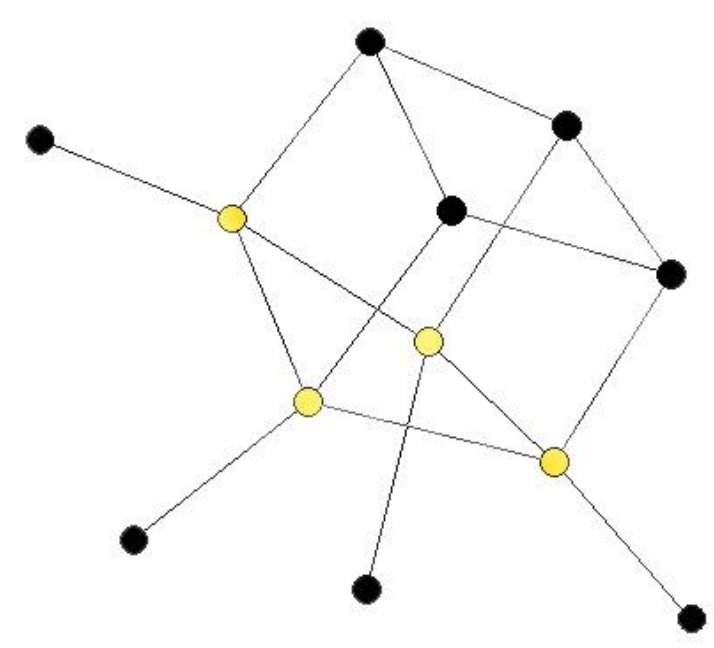

$\mathbf{W}_{4}$

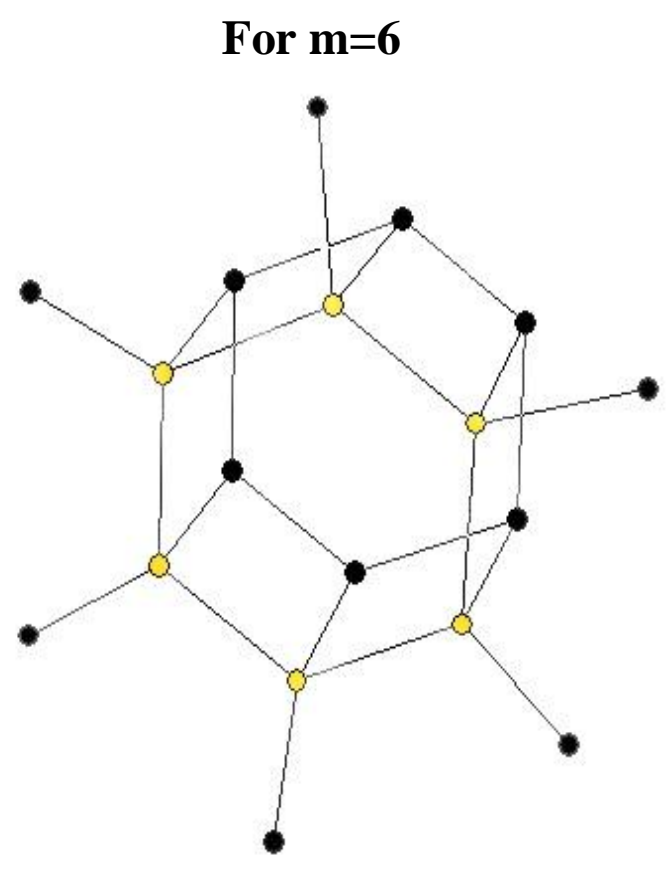

$W_{6}$

Fig.(10) 
The domination number of Levi graph $G_{1} \cup G_{2}$ :

By the program below we demonstrate how to find the domination number of $G_{1} \cup G_{2}$ using matlab :

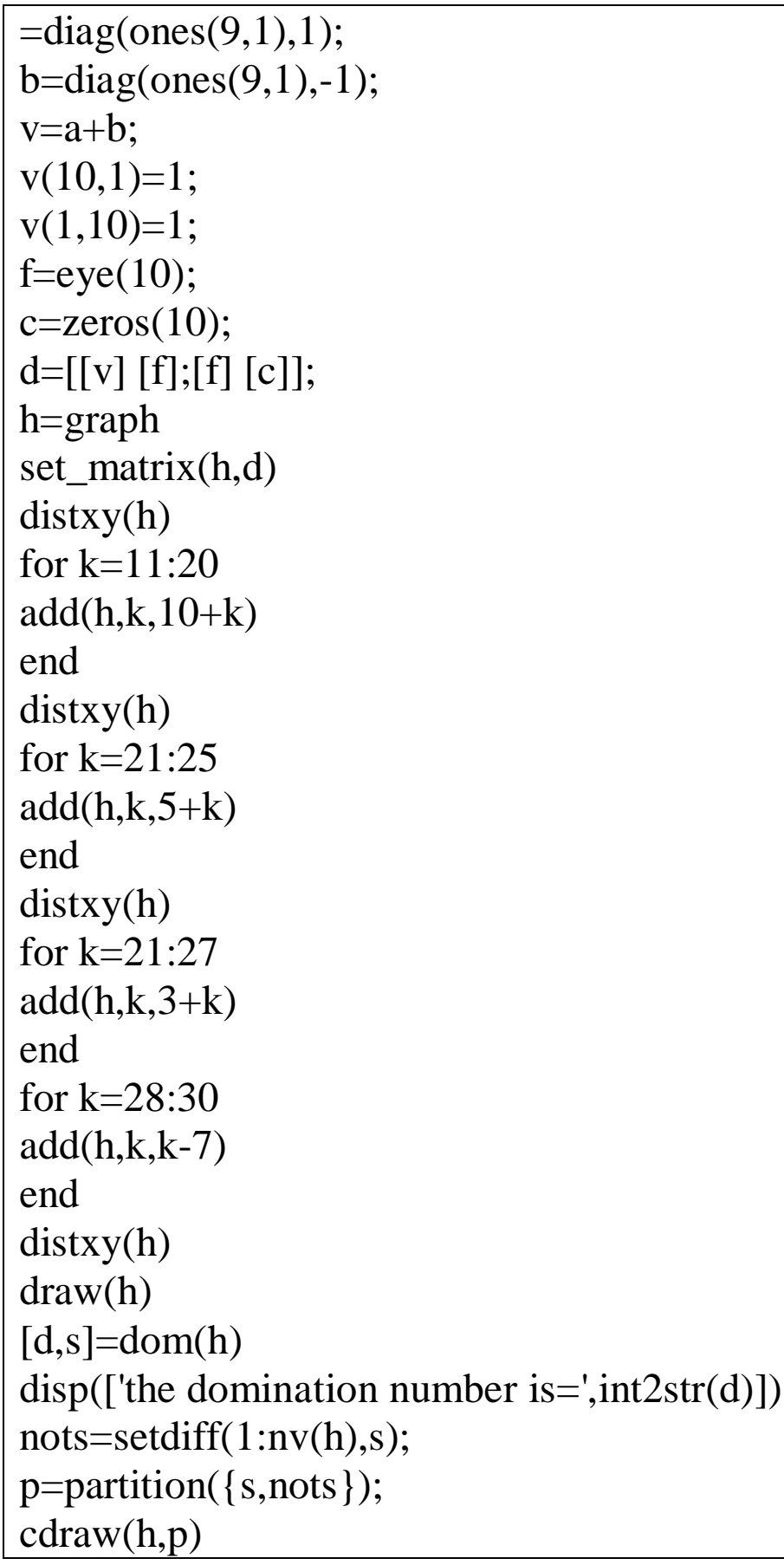

And Fig.(11) explain the particular results of the domination number of $G_{1}, G_{2}$ and Levi graph 

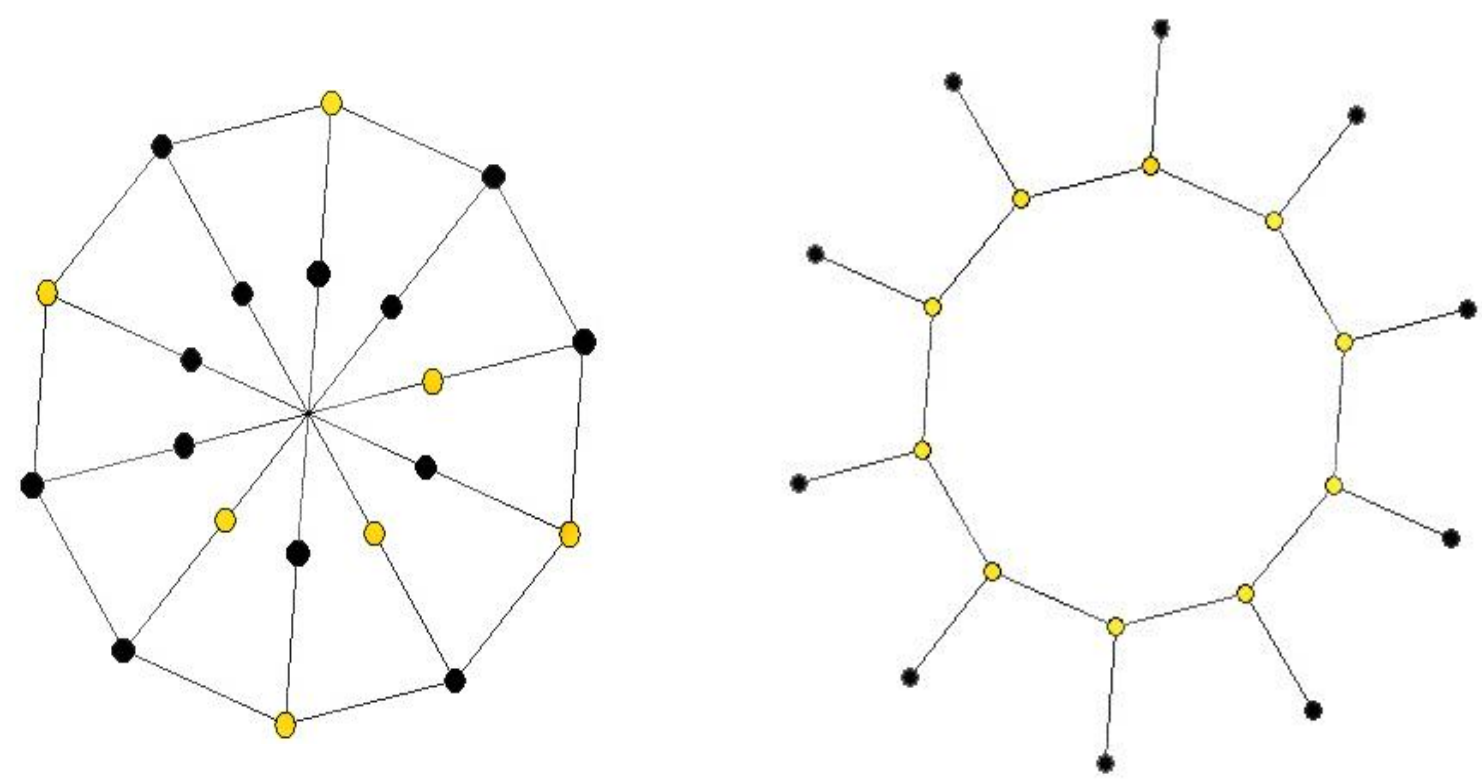

$\mathbf{G}_{1}$

$\mathbf{G}_{2}$

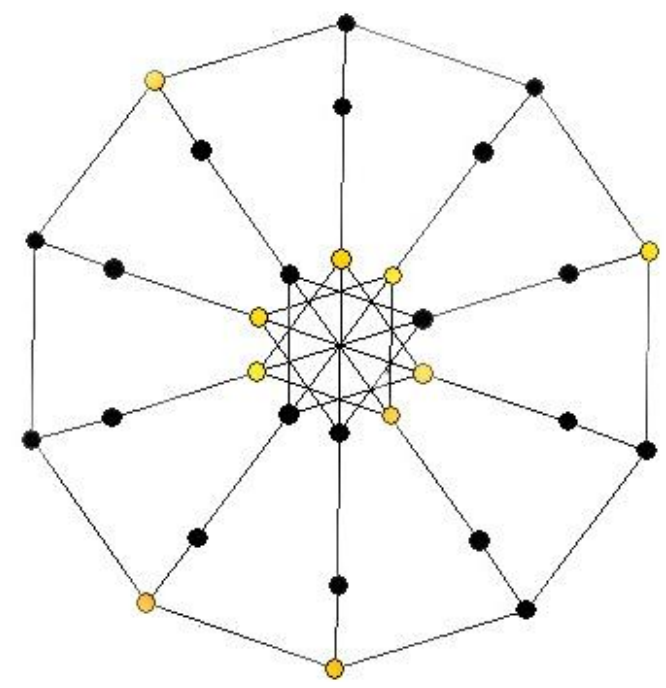

$G_{1} \cup G_{2}=$ Levi graph

Fig.(11) 


\section{Reference:}

1) Alithani, S. Peng, Y. H. and Atan, K. A. M. "on the domination number of some graphs" Int. Math. Forum, Vol. 3, (2008), No. 3740, pp.1879-1884.

2) Chartrand, G. and Lesniak, L. "Graphs and Digraphs", third addition,Chapman and Hall,(1996), chapter 10.

3) Haynes, T. W.; Hedetniemi, S. T. and Slater, P. J. "Fundamentals of Domination in Graphs", Marcel Dekker, New York-Basel-Hong Kong, 1998.

4) Hedetniemi, S. T. and Laskar, R. C. "Topics on domination", North Holland (February 15, 1991).

5) Hurink, J. L. and Nieberg, T. "Approximating minimum independent dominating sets in wireless networks", Information processing letters, Vol. 109,( 2008), pp.155-160

6) Kulli, V. R. and Janakiram, B. "The Nonsplit Domination Number Of A Graph", Indian J. Pure Appl. Math. Vol.31, (2000) No.5, pp.545550

7) Vuki, D. and Klobu, A. "K-dominating sets on linear benzenoids and on the infinite hexagonal grid", Croatica Chemica Acta.Vol.80 (2007), No.2, pp.187-191.

8) Zmazek, B. and Žerovnik, J., "On domination numbers of graph bundles", Instiute of mathematics,physics \& mechanics, Preprint series, Vol. 43 (2005), pp.1-10 .

9) Available at : http://mathworld.wolfram.com/HelmGraph.html.

10) Available at : http://mathworld.wolfram.com/WebGraph.html.

11) Available at : http://mathworld.wolfram.com/LeviGraph.html. 